\title{
Metal Dissipation and Inefficient Recycling Intensify Climate Forcing
}

\author{
Luca Ciacci $^{1,2, *}$, E.M. Harper ${ }^{1}$, N.T. Nassar ${ }^{1,3}$, Barbara K. Reck ${ }^{1}$, T.E. Graedel ${ }^{1}$ \\ ${ }^{1}$ Center for Industrial Ecology, School of Forestry \& Environmental Studies, Yale University, 195 \\ Prospect Street, New Haven (CT), United States \\ ${ }^{2}$ Interdepartmental Centre for Industrial Research “Energy \& Environment”, University of Bologna, Via \\ Angherà 22, Rimini, Italy \\ ${ }^{3}$ U.S. Geological Survey, Reston (VA), United States \\ ${ }^{*}$ Corresponding author. Tel.: +39-0541-434483; Fax: +39-0541-434480 \\ E-mail address: 1uca.ciacci5@unibo.it
}

Supporting Information 


\section{$\underline{\text { List of contents }}$}

S1.1 Calculations applied in the study (with an example for copper). p. S3

Table S1. Estimates of annual losses and embodied energy results for copper. p. S4

Table S2. Absolute global metal losses during use (in-use dissipation) and after use p. S5 (inefficient processing at end-of-life) estimated for 1993, 1998, 2003, and 2008.

Table S3. Comparison between the results of this study and global metal losses reported in p. S6 literature.

Table S4. Main end-use applications of metals, principal form commonly employed and p. S7 form considered in the analysis on the basis of existing life cycle inventories, average lifetime and standard deviation, distribution types, and end-of-life recycling rates (EOL-RR).

Table S5. Summary table of average, minimum, and maximum Gross Energy Requirement p. S23 (GER) and Global Warming Potential (GWP) values for each metal form considered in the analysis. Modified from Nuss and Eckelman (2014)

$\begin{array}{ll}\text { Table S6. Uncertainty ranges applied for sensitivity analysis. } & \text { p. S26 }\end{array}$

Figure S1. Uncertainty ranges (in grey) of the results for each use evaluated. p. S27 


\section{S1.1 Calculations applied in the study (with an example for copper)}

The following equation was applied to compute the energy required to provide the same amount of metal loss by deriving it from primary (virgin) raw materials for each year of the analysis:

$$
E_{i}=G E R_{i} \cdot \sum_{i}^{n}\left(m_{i}^{d}+m_{i}^{u}+m_{i}^{r}\right)
$$

Where $E_{i}$ is the embodied energy associated to metal losses for a given end-use $i$; GER $R_{i}$ is the gross energy requirement embodied with the most common metal form employed in the end-use $i$ (see Table S4 and S5); $m_{i}^{d}$ is the amount of lost metal due to in-use dissipation; $m_{i}^{u}$ is the amount of losses due to the employment of a metal in applications currently unrecyclable; $m_{i}^{r}$ is the amount of lost metal during recycling operations.

The total amount of embodied energy in lost flows $\left(E_{P}\right)$ for a metal is computed as sum of the embodied energy associated to each end-use for that specific metal, i.e.,

$$
E_{P}=\sum_{i}^{n} E_{i}
$$

$E_{P}$ provides the upper limit to permissible energy use by recyclers. However, because energy would need to be provided to process a recycled metal, $E_{P}$ constitutes the most extreme and optimistic estimate of potential gains associated to the recovery of a metal's losses. For several metals the energy required to process and remelt secondary metal sources $\left(E_{S}\right)$ is reported in literature. Where possible $E_{S}$ was applied to enable an estimate of the net potential for energy savings $\left(E_{N e t}\right)$ embodied in metal losses as follows:

$$
E_{N e t}=E_{P}-E_{S}
$$

To compute the greenhouse gas emissions embodied in metal losses and the associated potential savings, the GWP values reported in Table S5 have replaced GER values in the equations. The same equations were applied using minimum and maximum values for metal losses, GER, and GWP in the sensitivity analysis.

In the case of copper, estimates of losses due to in-use dissipation, losses due to a metal utilization in enduses currently unrecyclable, and losses due to recycling inefficiency are reported in Table S1. Perkilogram GER of primary in-use forms of copper (reported in Table S5) were multiplied by the annual amount of total losses in those forms. Copper's $E_{P}$ amounts at about $2.8 \mathrm{E}+11 \mathrm{MJ}$; because $E_{S}$ is commonly estimated to correspond at 0.14 of $E_{P},{ }^{1}$ the net potential for energy gains associated to copper losses results at $\sim 2.4 \mathrm{E}+11 \mathrm{MJ}$. This value corresponds at about $22 \%$ of the total energy required in 2008 for primary copper production $(1062 \mathrm{PJ}){ }^{2}$ 
Table S1. Estimates of copper losses (in kilograms) due to in-use dissipation, losses due to copper utilization in end-uses currently unrecyclable, losses due to recycling inefficiency, total losses, and the associated embodied energy (in MJ) in 2008.

$\begin{array}{lccccc}\text { End use } & \text { In-use dissipation } & \text { Currently unrecyclable } & \text { Recycling inefficiency } & \text { Total losses } & \text { E } \boldsymbol{i}[\mathbf{M J}] \\ \text { On-site waste } & 0.0 \mathrm{E}+00 & 0.0 \mathrm{E}+00 & 2.2 \mathrm{E}+08 & 2.2 \mathrm{E}+08 & 5.3 \mathrm{E}+10 \\ \text { Plumbing } & 1.1 \mathrm{E}+07 & 0.0 \mathrm{E}+00 & 1.0 \mathrm{E}+08 & 1.1 \mathrm{E}+08 & 5.7 \mathrm{E}+10 \\ \text { Wiring } & 0.0 \mathrm{E}+00 & 0.0 \mathrm{E}+00 & 5.4 \mathrm{E}+08 & 5.4 \mathrm{E}+08 & 6.6 \mathrm{E}+09 \\ \text { Built-in appliances } & 0.0 \mathrm{E}+00 & 0.0 \mathrm{E}+00 & 5.0 \mathrm{E}+08 & 5.0 \mathrm{E}+08 & 3.2 \mathrm{E}+10 \\ \text { Industrial EEE } & 0.0 \mathrm{E}+00 & 0.0 \mathrm{E}+00 & 9.0 \mathrm{E}+08 & 9.0 \mathrm{E}+08 & 2.7 \mathrm{E}+10 \\ \text { Consumer EEE } & 0.0 \mathrm{E}+00 & 0.0 \mathrm{E}+00 & 9.7 \mathrm{E}+08 & 9.7 \mathrm{E}+08 & 4.1 \mathrm{E}+10 \\ \text { Infrastructure } & 0.0 \mathrm{E}+00 & 0.0 \mathrm{E}+00 & 4.5 \mathrm{E}+08 & 4.5 \mathrm{E}+08 & 1.9 \mathrm{E}+10 \\ \text { Motor vehicles } & 4.6 \mathrm{E}+06 & 0.0 \mathrm{E}+00 & 6.9 \mathrm{E}+08 & 6.9 \mathrm{E}+08 & 1.3 \mathrm{E}+10 \\ \text { Other transport } & 0.0 \mathrm{E}+00 & 0.0 \mathrm{E}+00 & 3.2 \mathrm{E}+08 & 3.2 \mathrm{E}+08 & 2.9 \mathrm{E}+10\end{array}$

EEE - Electrical and electronic equipment 
Table S2. Absolute global metal losses during use (in-use dissipation) and after use (inefficient processing at end-of-life) estimated for 1993, 1998, 2003, and 2008. Values are in kilograms.

\begin{tabular}{|c|c|c|c|c|c|c|c|c|c|}
\hline Element & 1993 & 1998 & 2003 & 2008 & Element & 1993 & 1998 & 2003 & 2008 \\
\hline Aluminum & $7.2 \mathrm{E}+09$ & $8.5 \mathrm{E}+09$ & $1.0 \mathrm{E}+10$ & $1.3 \mathrm{E}+10$ & Tin & $1.8 \mathrm{E}+08$ & $1.9 \mathrm{E}+08$ & $1.9 \mathrm{E}+08$ & $2.1 \mathrm{E}+08$ \\
\hline Scandium & $2.5 \mathrm{E}+02$ & $3.9 \mathrm{E}+02$ & $5.7 \mathrm{E}+02$ & $7.4 \mathrm{E}+02$ & Antimony & $5.2 \mathrm{E}+07$ & $7.2 \mathrm{E}+07$ & $1.0 \mathrm{E}+08$ & $1.3 \mathrm{E}+08$ \\
\hline Titanium & $1.6 \mathrm{E}+09$ & $1.8 \mathrm{E}+09$ & $2.0 \mathrm{E}+09$ & $2.3 \mathrm{E}+09$ & Tellurium & $1.5 \mathrm{E}+05$ & $2.0 \mathrm{E}+05$ & $2.1 \mathrm{E}+05$ & $2.6 \mathrm{E}+05$ \\
\hline Vanadium & $8.9 \mathrm{E}+06$ & $1.1 \mathrm{E}+07$ & $1.3 \mathrm{E}+07$ & $1.4 \mathrm{E}+07$ & Lanthanum & * & $7.8 \mathrm{E}+06$ & $1.6 \mathrm{E}+07$ & $1.7 \mathrm{E}+07$ \\
\hline Chromium & $2.8 \mathrm{E}+09$ & $3.4 \mathrm{E}+09$ & $4.1 \mathrm{E}+09$ & $4.8 \mathrm{E}+09$ & Cerium & $*$ & $8.5 \mathrm{E}+06$ & $1.6 \mathrm{E}+07$ & $1.8 \mathrm{E}+07$ \\
\hline Manganese & $3.2 \mathrm{E}+09$ & $3.7 \mathrm{E}+09$ & $4.2 \mathrm{E}+09$ & $4.9 \mathrm{E}+09$ & Praseodymium & $*$ & $2.7 \mathrm{E}+05$ & $4.7 \mathrm{E}+05$ & $7.4 \mathrm{E}+05$ \\
\hline Iron & $8.5 \mathrm{E}+10$ & $9.6 \mathrm{E}+10$ & $1.1 \mathrm{E}+11$ & $1.2 \mathrm{E}+11$ & Neodymium & $*$ & $8.9 \mathrm{E}+05$ & $2.2 \mathrm{E}+06$ & $3.8 \mathrm{E}+06$ \\
\hline Cobalt & $2.8 \mathrm{E}+07$ & $2.5 \mathrm{E}+07$ & $3.8 \mathrm{E}+07$ & $5.6 \mathrm{E}+07$ & Samarium & * & $5.2 \mathrm{E}+05$ & $1.2 \mathrm{E}+06$ & $1.6 \mathrm{E}+06$ \\
\hline Nickel & $2.0 \mathrm{E}+08$ & $2.3 \mathrm{E}+08$ & $2.6 \mathrm{E}+08$ & $2.7 \mathrm{E}+08$ & Europium & $*$ & $7.6 \mathrm{E}+04$ & $1.8 \mathrm{E}+05$ & $2.7 \mathrm{E}+05$ \\
\hline Copper & $3.1 \mathrm{E}+09$ & $3.6 \mathrm{E}+09$ & $4.1 \mathrm{E}+09$ & $4.7 \mathrm{E}+09$ & Gadolinium & $*$ & $1.7 \mathrm{E}+05$ & $3.7 \mathrm{E}+05$ & $5.8 \mathrm{E}+05$ \\
\hline Zinc & $4.0 \mathrm{E}+09$ & $4.4 \mathrm{E}+09$ & $4.9 \mathrm{E}+09$ & $5.6 \mathrm{E}+09$ & Terbium & * & $4.8 \mathrm{E}+04$ & $1.2 \mathrm{E}+05$ & $1.8 \mathrm{E}+05$ \\
\hline Gallium & $1.9 \mathrm{E}+01$ & $2.1 \mathrm{E}+01$ & $3.5 \mathrm{E}+01$ & $5.5 \mathrm{E}+01$ & Dysprosium & $*$ & $5.7 \mathrm{E}+02$ & $1.2 \mathrm{E}+04$ & $9.8 \mathrm{E}+04$ \\
\hline Germanium & $8.1 \mathrm{E}+04$ & $7.0 \mathrm{E}+04$ & $7.2 \mathrm{E}+04$ & $1.2 \mathrm{E}+05$ & Hafnium & $3.9 \mathrm{E}+04$ & $4.4 \mathrm{E}+04$ & $4.8 \mathrm{E}+04$ & $6.6 \mathrm{E}+04$ \\
\hline Arsenic & $1.4 \mathrm{E}+07$ & $1.5 \mathrm{E}+07$ & $2.3 \mathrm{E}+07$ & $3.7 \mathrm{E}+07$ & Tantalum & $3.5 \mathrm{E}+05$ & $5.0 \mathrm{E}+05$ & $7.8 \mathrm{E}+05$ & $1.2 \mathrm{E}+06$ \\
\hline Selenium & $1.1 \mathrm{E}+06$ & $1.2 \mathrm{E}+06$ & $1.1 \mathrm{E}+06$ & $1.3 \mathrm{E}+06$ & Tungsten & $4.7 \mathrm{E}+07$ & $4.1 \mathrm{E}+07$ & $5.3 \mathrm{E}+07$ & $8.0 \mathrm{E}+07$ \\
\hline Yttrium & $0.0 \mathrm{E}+00$ & $1.9 \mathrm{E}+06$ & $5.1 \mathrm{E}+06$ & $7.8 \mathrm{E}+06$ & Rhenium & $2.0 \mathrm{E}+04$ & $2.0 \mathrm{E}+04$ & $2.9 \mathrm{E}+04$ & $3.6 \mathrm{E}+04$ \\
\hline Zirconium & $3.8 \mathrm{E}+05$ & $3.3 \mathrm{E}+05$ & $3.5 \mathrm{E}+05$ & $4.6 \mathrm{E}+05$ & Iridium & $6.1 \mathrm{E}+02$ & $1.4 \mathrm{E}+03$ & $2.1 \mathrm{E}+03$ & $3.1 \mathrm{E}+03$ \\
\hline Niobium & $4.6 \mathrm{E}+06$ & $6.3 \mathrm{E}+06$ & $8.4 \mathrm{E}+06$ & $1.2 \mathrm{E}+07$ & Platinum & $2.4 \mathrm{E}+04$ & $3.0 \mathrm{E}+04$ & $3.7 \mathrm{E}+04$ & $5.0 \mathrm{E}+04$ \\
\hline Molybdenum & $6.5 \mathrm{E}+07$ & $7.7 \mathrm{E}+07$ & $7.7 \mathrm{E}+07$ & $8.7 \mathrm{E}+07$ & Gold & $1.4 \mathrm{E}+05$ & $1.6 \mathrm{E}+05$ & $1.8 \mathrm{E}+05$ & $2.1 \mathrm{E}+05$ \\
\hline Ruthenium & $2.1 \mathrm{E}+03$ & $3.4 \mathrm{E}+03$ & $5.1 \mathrm{E}+03$ & $7.5 \mathrm{E}+03$ & Mercury & $4.1 \mathrm{E}+06$ & $3.2 \mathrm{E}+06$ & $2.4 \mathrm{E}+06$ & $2.0 \mathrm{E}+06$ \\
\hline Rhodium & $3.4 \mathrm{E}+03$ & $4.6 \mathrm{E}+03$ & $6.3 \mathrm{E}+03$ & $8.5 \mathrm{E}+03$ & Thallium & $1.2 \mathrm{E}+04$ & $1.2 \mathrm{E}+04$ & $1.0 \mathrm{E}+04$ & $1.1 \mathrm{E}+04$ \\
\hline Palladium & $7.3 \mathrm{E}+04$ & $9.6 \mathrm{E}+04$ & $1.1 \mathrm{E}+05$ & $1.1 \mathrm{E}+05$ & Lead & $3.0 \mathrm{E}+09$ & $3.2 \mathrm{E}+09$ & $3.5 \mathrm{E}+09$ & $4.0 \mathrm{E}+09$ \\
\hline Silver & $1.5 \mathrm{E}+07$ & $1.5 \mathrm{E}+07$ & $1.7 \mathrm{E}+07$ & $1.9 \mathrm{E}+07$ & Bismuth & $3.8 \mathrm{E}+06$ & $3.5 \mathrm{E}+06$ & $3.9 \mathrm{E}+06$ & $4.0 \mathrm{E}+06$ \\
\hline Cadmium & $2.1 \mathrm{E}+07$ & $1.6 \mathrm{E}+07$ & $1.6 \mathrm{E}+07$ & $1.4 \mathrm{E}+07$ & Thorium & $1.4 \mathrm{E}+05$ & $8.1 \mathrm{E}+04$ & $7.8 \mathrm{E}+04$ & $9.9 \mathrm{E}+04$ \\
\hline Indium & $7.3 \mathrm{E}+04$ & $7.8 \mathrm{E}+04$ & $1.1 \mathrm{E}+05$ & $1.7 \mathrm{E}+05$ & Uranium & $6.8 \mathrm{E}+07$ & $7.3 \mathrm{E}+07$ & $7.6 \mathrm{E}+07$ & $7.4 \mathrm{E}+07$ \\
\hline
\end{tabular}


1 Table S3. Comparison between the results of this study and global metal losses reported in 2 literature.

\begin{tabular}{lccccc} 
& & & \multicolumn{3}{c}{ Previous estimates } \\
\cline { 4 - 6 } Element & Unit & 2008 estimate & Value & Year & Source \\
Aluminum & Tg/year & 13.2 & 10.0 & 2009 & {$[3]$} \\
Iron & Tg/year & 120.0 & 93.0 & 2000 & {$[4]$} \\
Copper & Tg/year & 4.7 & 6.5 & 2010 & {$[5]$} \\
Selenium & Gg/year & 1.3 & 2.1 & 2010 & {$[6]$} \\
Lead & Tg/year & 4.0 & 2.0 & 2000 & {$[7]$} \\
Tellurium & Gg/year & 0.3 & 0.1 & 2010 & {$[8]$}
\end{tabular}

31 gigagram $(\mathrm{Gg})=10^{9}$ grams

41 teragram $(\mathrm{Tg})=10^{12}$ grams

5

6

7 
Table S4. Main end-use applications of metals, principal form commonly employed and form considered in the analysis on the basis of

2 existing life cycle inventories, average lifetime and standard deviation, distribution types, and end-of-life recycling rates (EOL-RR). For

3 more details see the cited references.

\begin{tabular}{|c|c|c|c|c|c|c|c|}
\hline 를 & End-use & Principal form & $\begin{array}{l}\text { Form considered in } \\
\text { the analysis }\end{array}$ & $\begin{array}{l}\text { Lifetime (years): } \\
\text { average } \pm \text { standard } \\
\text { deviation }\end{array}$ & Distribution type & EOL-RR & Reference \\
\hline \multirow{9}{*}{ 志 } & Transportation & Metal & Metal & $20 \pm 1.5$ & Normal & $75 \%$ & [9] \\
\hline & Building \& construction & Metal & Metal & $55 \pm 10$ & Normal & $70 \%$ & [9] \\
\hline & Packaging & Metal & Metal & $1 \pm 0.1$ & Normal & $60 \%$ & [9] \\
\hline & Electrical & Metal & Metal & $40 \pm 2.5$ & Normal & $50 \%$ & [9] \\
\hline & Machinery & Metal & Metal & $25 \pm 2.5$ & Normal & $45 \%$ & [9] \\
\hline & Consumer durables & Metal & Metal & $15 \pm 2$ & Normal & $20 \%$ & [9] \\
\hline & Other & Metal & Metal & - & - & - & - \\
\hline & Deoxidation & Metal & Metal & $1 \pm 0.1$ & Normal & $0 \%$ & $\begin{array}{l}\text { Own assessment } \\
\text { based on [10] }\end{array}$ \\
\hline & Miscellaneous & Mixed & Metal & $12 \pm 2$ & Normal & $20 \%$ & [9] \\
\hline \multirow{4}{*}{ 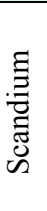 } & Aerospace and defense & Metal & Scandium oxide & $25 \pm 3.5$ & Weibull & $<1 \%$ & {$[11]$} \\
\hline & Lighting & Scandium oxide & Scandium oxide & $2.50 \pm 0.25$ & Normal & $<1 \%$ & {$[11]$} \\
\hline & Sports equipment & Metal & Scandium oxide & $14.7 \pm 1.75$ & Weibull & $<1 \%$ & {$[11]$} \\
\hline & Other & Mixed & Scandium oxide & $5 \pm 0.5$ & Normal & $<1 \%$ & {$[11]$} \\
\hline \multirow{5}{*}{.气 } & Pigments (paint) & Titanium dioxide & Titanium dioxide & $20 \pm 3.5$ & Weibull & $0 \%$ & [9] \\
\hline & Pigments (plastic) & Titanium dioxide & Titanium dioxide & $15 \pm 3.5$ & Weibull & $0 \%$ & [9] \\
\hline & Pigments (paper) & Titanium dioxide & Titanium dioxide & $5 \pm 3.5$ & Weibull & $0 \%$ & [9] \\
\hline & Pigments (other) & Titanium dioxide & Titanium dioxide & $5 \pm 3.5$ & Weibull & $0 \%$ & [9] \\
\hline & Other (metallurgy) & Metal; oxides & Metal & $20 \pm 3.5$ & Weibull & $>50 \%$ & [9] \\
\hline
\end{tabular}




\begin{tabular}{|c|c|c|c|c|c|c|c|}
\hline 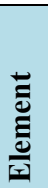 & End-use & Principal form & $\begin{array}{l}\text { Form considered in } \\
\text { the analysis }\end{array}$ & $\begin{array}{l}\text { Lifetime (years): } \\
\text { average } \pm \text { standard } \\
\text { deviation }\end{array}$ & Distribution type & EOL-RR & Reference \\
\hline \multirow{8}{*}{ 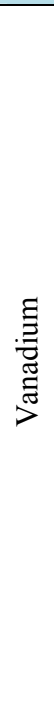 } & Transportation & Metal & Metal & $20 \pm 6$ & Normal & $53 \%$ & {$[12]$} \\
\hline & $\begin{array}{l}\text { Machinery: metalworking and } \\
\text { tools }\end{array}$ & Metal & Metal & $30 \pm 9$ & Normal & $53 \%$ & {$[12]$} \\
\hline & Machinery: other and equipment & Metal & Metal & $30 \pm 9$ & Normal & $53 \%$ & {$[12]$} \\
\hline & $\begin{array}{c}\text { Building and heavy } \\
\text { infrastructure }\end{array}$ & Metal & Metal & $75 \pm 22.5$ & Normal & $53 \%$ & {$[12]$} \\
\hline & Oil and gas industry & Metal & Metal & $60 \pm 18$ & Normal & $53 \%$ & {$[12]$} \\
\hline & $\begin{array}{l}\text { Mining, quarrying and } \\
\text { lumbering }\end{array}$ & Metal & Metal & $20 \pm 6$ & Normal & $53 \%$ & {$[12]$} \\
\hline & $\begin{array}{l}\text { Chemicals (maleic anhydride } \\
\text { and sulfuric acid } \\
\text { production) }\end{array}$ & Metal & Metal & $8 \pm 2.4$ & Normal & $53 \%$ & {$[12]$} \\
\hline & Other & Metal & Metal & $15 \pm 4.5$ & Normal & $53 \%$ & {$[12]$} \\
\hline \multirow{8}{*}{ 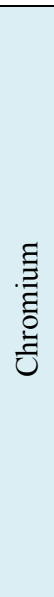 } & Building and infrastructure & Metal & Metal & $70 \pm 21$ & & $54 \%$ & {$[13]$} \\
\hline & Industrial machinery & Metal & Metal & $25 \pm 7.5$ & & $54 \%$ & {$[13]$} \\
\hline & Transportation & Metal & Metal & $13.5 \pm 4.05$ & & $54 \%$ & {$[13]$} \\
\hline & $\begin{array}{l}\text { Household appliances and } \\
\text { electronics }\end{array}$ & Metal & Metal & $12.5 \pm 3.75$ & & $54 \%$ & {$[13]$} \\
\hline & Other & Mixed & Metal & - & & - & \\
\hline & Refractory and foundries & Chromite; mag-chrome & Chromite & $18.6 \pm 1.92$ & & $0 \%$ & {$[14]$} \\
\hline & Chemicals & $\begin{array}{l}\text { Mixed; Chromium oxides; } \\
\text { chromated copper } \\
\text { arsenate; } \mathrm{PbCrO}_{4}\end{array}$ & Sodium dichromate & $18.6 \pm 1.92$ & & $0 \%$ & {$[14]$} \\
\hline & Other metallurgy & Metal & Metal & $18.6 \pm 1.92$ & & $54 \%$ & {$[13]$} \\
\hline
\end{tabular}




\begin{tabular}{|c|c|c|c|c|c|c|c|}
\hline 를 & End-use & Principal form & $\begin{array}{l}\text { Form considered in } \\
\text { the analysis }\end{array}$ & $\begin{array}{l}\text { Lifetime (years): } \\
\text { average } \pm \text { standard } \\
\text { deviation }\end{array}$ & Distribution type & EOL-RR & Reference \\
\hline \multirow{6}{*}{ 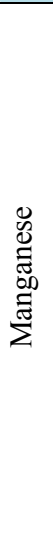 } & Construction & Metal & Metal & $75 \pm 22.5$ & & $53 \%$ & {$[13]$} \\
\hline & Machinery & Metal & Metal & $30 \pm 9$ & & $53 \%$ & {$[13]$} \\
\hline & Transportation & Metal & Metal & $20 \pm 6$ & & $53 \%$ & {$[13]$} \\
\hline & Chemicals & $\begin{array}{l}\text { Mixed; potassium } \\
\text { permanganate; maneb; } \\
\text { manganese oxides; } \\
\text { manganese carbonates }\end{array}$ & Manganese oxide & $1 \pm 0.3$ & & $0 \%$ & {$[13]$} \\
\hline & Batteries & Manganese dioxide & Manganese oxide & $1 \pm 0.3$ & & $53 \%$ & $\begin{array}{l}\text { Own assessment } \\
\text { based on [14] }\end{array}$ \\
\hline & Other iron and steel & Metal & Metal & $19 \pm 5.7$ & & $53 \%$ & {$[13]$} \\
\hline \multirow{4}{*}{$\stackrel{0}{\Xi}$} & Construction & Metal & Metal & $75 \pm 22.5$ & Normal & $82 \%$ & {$[13]$} \\
\hline & Machinery & Metal & Metal & $20 \pm 6$ & Normal & $82 \%$ & {$[13]$} \\
\hline & Transportation & Metal & Metal & $30 \pm 9$ & Normal & $87 \%$ & {$[13]$} \\
\hline & Metal products & Metal & Metal & $15 \pm 4.5$ & Normal & $58 \%$ & [13] \\
\hline \multirow{6}{*}{$\begin{array}{l}\frac{1}{\pi} \\
0 \\
0\end{array}$} & Superalloys & Metal & Metal & $5 \pm 0.5$ & Normal & $80 \%$ & [9] \\
\hline & Cemented carbides (cuttings) & Metal & Metal & $1 \pm 0.1$ & Normal & $15 \%$ & {$[9]$} \\
\hline & Cemented carbides (dies) & Metal & Metal & $1 \pm 0.1$ & Normal & $45 \%$ & [9] \\
\hline & $\begin{array}{l}\text { Cemented carbides (mining and } \\
\text { construction) }\end{array}$ & Metal & Metal & $1 \pm 0.1$ & Normal & $5 \%$ & [9] \\
\hline & Magnets & Metal & Metal & $5 \pm 0.5$ & Normal & $5 \%$ & [9] \\
\hline & Catalysts (polyester) & $\begin{array}{l}\text { Cobalt carboxylates; } \\
\text { cobalt oxides; cobalt } \\
\text { fluoride; cobalt sulphide }\end{array}$ & Metal $^{1}$ & $8 \pm 3.5$ & Normal & $89 \%$ & [9] \\
\hline
\end{tabular}

\footnotetext{
${ }^{1}$ Cobalt oxides are produced by controlled oxidation of the metal $\left(\mathrm{T}>900{ }^{\circ} \mathrm{C}\right)$ followed by cooling in protective atmosphere to prevent partial oxidation [15]
} 


\begin{tabular}{|c|c|c|c|c|c|c|c|}
\hline 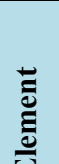 & End-use & Principal form & $\begin{array}{l}\text { Form considered in } \\
\text { the analysis }\end{array}$ & $\begin{array}{l}\text { Lifetime (years): } \\
\text { average } \pm \text { standard } \\
\text { deviation }\end{array}$ & Distribution type & EOL-RR & Reference \\
\hline & Catalyst (petroleum refining) & $\begin{array}{l}\text { Cobalt oxides, cobalt } \\
\text { aluminate; cobalt (II) } \\
\text { chromate; cobalt chloride }\end{array}$ & Metal $^{1}$ & $2 \pm 0.2$ & Normal & $0 \%$ & {$[9]$} \\
\hline & Batteries (consumer) & Metal & Metal & $2.5 \pm 0.25$ & Normal & $10 \%$ & [9] \\
\hline & Batteries (hybrid vehicles) & Metal & Metal & $8 \pm 3.5$ & Weibull & $89 \%$ & [9] \\
\hline & Other & Cobalamin; mixed & Metal $^{1}$ & $5 \pm 0.5$ & Normal & $0 \%$ & [9] \\
\hline \multirow{6}{*}{ 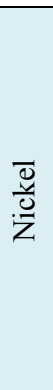 } & Building and infrastructure & Metal & Metal & $50 \pm 15$ & Normal & $87 \%$ & [9] \\
\hline & Transportation: automotive & Metal & Metal & $14 \pm 2.1$ & Normal & $74 \%$ & {$[9]$} \\
\hline & Transportation: nonautomotive & Metal & Metal & $30 \pm 6$ & Normal & $74 \%$ & {$[9]$} \\
\hline & Industrial machinery & Metal & Metal & $25 \pm 5$ & Normal & $87 \%$ & [9] \\
\hline & $\begin{array}{l}\text { Household appliances and } \\
\text { electronics }\end{array}$ & Metal & Metal & $15 \pm 3$ & Normal & $29 \%$ & {$[9]$} \\
\hline & Metal goods & Metal & Metal & $15 \pm 3.75$ & Normal & $48 \%$ & [9] \\
\hline \multirow{9}{*}{$\frac{\overline{0}}{2}$} & On-site waste & Metal & Metal & $1 \pm 4$ & Weibull & $72 \%$ & {$[16]$} \\
\hline & Plumbing & Metal & Metal & $40 \pm 4$ & Weibull & $82 \%$ & {$[16]$} \\
\hline & Wiring & Metal & Metal & $25 \pm 4$ & Weibull & $61 \%$ & {$[16]$} \\
\hline & Built-in appliances & Metal & Metal & $17 \pm 4$ & Weibull & $66 \%$ & {$[16]$} \\
\hline & $\begin{array}{l}\text { Industrial electrical and } \\
\text { electronic equipment }\end{array}$ & Metal & Metal & $20 \pm 4$ & Weibull & $60 \%$ & [16] \\
\hline & $\begin{array}{l}\text { Consumer electrical and } \\
\text { electronic equipment }\end{array}$ & Metal & Metal & $10 \pm 4$ & Weibull & $60 \%$ & [16] \\
\hline & Infrastructure & Metal & Metal & $50 \pm 4$ & Weibull & $60 \%$ & {$[16]$} \\
\hline & Motor vehicles & Metal & Metal & $10 \pm 4$ & Weibull & $60 \%$ & {$[16]$} \\
\hline & Other transport & Metal & Metal & $30 \pm 4$ & Weibull & $60 \%$ & {$[16]$} \\
\hline
\end{tabular}




\begin{tabular}{|c|c|c|c|c|c|c|c|}
\hline 흄 & End-use & Principal form & $\begin{array}{l}\text { Form considered in } \\
\text { the analysis }\end{array}$ & $\begin{array}{l}\text { Lifetime (years): } \\
\text { average } \pm \text { standard } \\
\text { deviation }\end{array}$ & Distribution type & EOL-RR & Reference \\
\hline \multirow{4}{*}{$\stackrel{\circlearrowright}{*}$} & Galvanizing & Metal & Metal & $17 \pm 3.5$ & Weibull & $37 \%$ & {$[17,18]$} \\
\hline & Zinc-based alloys & Metal & Metal & $16 \pm 3.5$ & Weibull & $37 \%$ & {$[17,18]$} \\
\hline & Brass and bronze & Metal & Metal & $19 \pm 3.5$ & Weibull & $37 \%$ & {$[17,18]$} \\
\hline & Other & Zinc oxide & Metal $^{2}$ & $13.5 \pm 1.81$ & Weibull & $0 \%$ & {$[17,18]$} \\
\hline \multirow{3}{*}{$\stackrel{\Xi}{\Xi}$} & Integrated circuits & $\begin{array}{l}\text { Gallium arsenide; gallium } \\
\text { nitride; gallium } \\
\text { antimonide }\end{array}$ & Metal & $13.4 \pm 2.15$ & Weibull & $<1 \%$ & {$[11]$} \\
\hline & Optoelectronic devices & $\begin{array}{l}\text { Gallium arsenide; gallium } \\
\text { nitride; gallium } \\
\text { antimonide }\end{array}$ & Metal & $20 \pm 3.5$ & Weibull & $<1 \%$ & {$[11]$} \\
\hline & Other & Mixed & Metal & $5 \pm 0.5$ & Weibull & $<1 \%$ & {$[11]$} \\
\hline \multirow{5}{*}{ 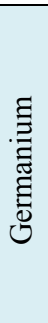 } & Fiber optic systems & Germanium dioxide & Metal $^{3}$ & $14.6 \pm 1.7$ & Weibull & $0 \%$ & {$[14,17]$} \\
\hline & Infrared optics & Germanium dioxide & $\mathrm{Metal}^{3}$ & $13.10 \pm 1.79$ & Weibull & $8 \%$ & {$[17]$} \\
\hline & Polymerization catalysts & Germanium dioxide & $\mathrm{Metal}^{3}$ & $1 \pm 0.1$ & Normal & $0 \%$ & {$[17]$} \\
\hline & $\begin{array}{l}\text { Electronic and solar electric } \\
\text { applications }\end{array}$ & Silicon-germanium alloys & Metal & $12.4 \pm 2.33$ & Weibull & $15 \%$ & {$[14,17]$} \\
\hline & Other & Mixed & Metal & $5 \pm 0.5$ & Normal & $0 \%$ & {$[17]$} \\
\hline \multirow{3}{*}{ 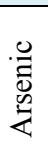 } & Batteries & Metal & Metal & $12.3 \pm 2.2$ & Weibull & $0 \%$ & {$[22]$} \\
\hline & Antifriction additive & Mixed; arsenic trioxide & Arsenic trioxide $^{4}$ & $13.5 \pm 1.81(15.2)$ & Weibull & $0 \%$ & {$[22]$} \\
\hline & Germanium-arsenic-selenium & Metal & Metal & $13.1 \pm 1.8(14.7)$ & Weibull & $0 \%$ & {$[22]$} \\
\hline
\end{tabular}

\footnotetext{
2 The industrial production of zinc oxide can follow different routes. In the indirect process, pure zinc is the starting material, which is heated and oxidized in air. Solutions of purified zinc are the starting input for wet chemical processes, from which zinc is obtained by precipitation as carbonate or hydroxide. The zinc oxide so produced is utilized as an intermediate product for chemicals production. [19]

3 The main production routes for germanium dioxide are the hydrolysis of germanium tetrachloride and the ignition of germanium disulfide. $\mathrm{GeCl} \mathrm{C}_{4}$ can be produced by the reaction of hydrochloric acid on concentrates of germanium oxides or germinate or by the reaction of chlorine with metallic germanium. [20]

${ }^{4}$ Arsenic trioxide is the sole starting material for applications in forestry, agriculture, industrial chemicals, and in the glass industry as fining agent and decolorizer. [21]
} 


\begin{tabular}{|c|c|c|c|c|c|c|c|}
\hline \multirow{6}{*}{ 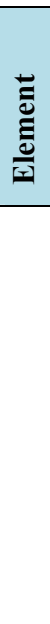 } & End-use & Principal form & $\begin{array}{l}\text { Form considered in } \\
\text { the analysis }\end{array}$ & $\begin{array}{l}\text { Lifetime (years): } \\
\text { average } \pm \text { standard } \\
\text { deviation }\end{array}$ & Distribution type & EOL-RR & Reference \\
\hline & optical materials & & & & & & \\
\hline & Semiconductors & Metal & Metal & $12.4 \pm 2.33(14.0)$ & Weibull & $0 \%$ & {$[22]$} \\
\hline & Pressure-treated wood & Mixed; arsenic trioxide & Arsenic trioxide & $30 \pm 3.5$ & Weibull & $0 \%$ & $\begin{array}{l}\text { Own assessment } \\
\text { based on [14] }\end{array}$ \\
\hline & Glass & Mixed; arsenic trioxide & Arsenic trioxide & $12.5 \pm 3.5$ & Weibull & $0 \%$ & $\begin{array}{l}\text { Own assessment } \\
\text { based on [14] }\end{array}$ \\
\hline & Agricultural chemicals & Mixed; arsenic trioxide & Arsenic trioxide & $1 \pm 0.1$ & Normal & $0 \%$ & $\begin{array}{l}\text { Own assessment } \\
\text { based on [14] }\end{array}$ \\
\hline \multirow{7}{*}{ 当 } & Rectifiers & Metal & Metal & $10 \pm 0.1$ & Normal & $50 \%$ & {$[14,22,23]$} \\
\hline & Photoreceptors & Metal & Metal & $6.5 \pm 2.2(7.3)$ & Weibull & $50 \%$ & {$[14,22,23]$} \\
\hline & Photovoltaic & Metal & Metal & $12.4 \pm 2.33(14.0)$ & Weibull & $0 \%$ & {$[14,22,23]$} \\
\hline & Glass manufacturing & $\begin{array}{l}\text { Sodium selenite; sodium } \\
\text { selenite }\end{array}$ & $\mathrm{Metal}^{5}$ & $10 \pm 3.5$ & Weibull & $0 \%$ & {$[14,22,23]$} \\
\hline & Chemicals and pigments & $\begin{array}{l}\text { Mixed; dialkylselenium } \\
\text { carbamates; cadmium } \\
\text { sulfoselenide }\end{array}$ & $\mathrm{Metal}^{5}$ & $5 \pm 3.5$ & Weibull & $0 \%$ & {$[14,22,23]$} \\
\hline & Biological & $\begin{array}{l}\text { Sodium selenite; sodium } \\
\text { selenite }\end{array}$ & $\mathrm{Metal}^{5}$ & $1 \pm 3.5$ & Weibull & $0 \%$ & {$[14,22,23]$} \\
\hline & Metallurgy & Metal & Metal & $20 \pm 3.5$ & Weibull & $0 \%$ & {$[14,22,23]$} \\
\hline \multirow{2}{*}{$\underset{\Xi}{\Xi}$} & Phosphors & $\begin{array}{l}\text { Yttrium oxide; yttrium } \\
\text { aluminum garnet }\end{array}$ & Yttrium oxide & $4 \pm 1$ & Normal & $0 \%$ & {$[25]$} \\
\hline & Ceramics & $\begin{array}{l}\text { Yttrium oxide; yttrium- } \\
\text { zirconia }\end{array}$ & Yttrium oxide & $5 \pm 2$ & Normal & $0 \%$ & {$[25]$} \\
\hline
\end{tabular}

${ }^{5}$ Sodium selenite and sodium selenate are formed by oxidation of selenium through selenous acid and selenic acid production, respectively. [24] 


\begin{tabular}{|c|c|c|c|c|c|c|c|}
\hline 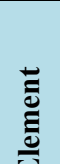 & End-use & Principal form & $\begin{array}{l}\text { Form considered in } \\
\text { the analysis }\end{array}$ & \begin{tabular}{|l|} 
Lifetime (years): \\
average \pm standard \\
deviation
\end{tabular} & Distribution type & EOL-RR & Reference \\
\hline & Other & $\begin{array}{l}\text { Mixed; yttrium oxide; } \\
\text { yttrium-90 }\end{array}$ & Yttrium oxide & $1 \pm 0.1$ & Normal & $0 \%$ & {$[25]$} \\
\hline \multirow{4}{*}{ 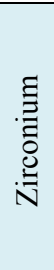 } & Ceramics & Zirconium silicate & Zirconium silicate & $10 \pm 3.5$ & Weibull & $<1 \%$ & {$[26]$} \\
\hline & Refractory & Zirconium silicate & Zirconium silicate & $2 \pm 0.2$ & Normal & $<1 \%$ & {$[26]$} \\
\hline & Foundry molds & Metal; zirconium silicate & Metal & $10.7 \pm 1.33$ & Weibull & $<1 \%$ & {$[26]$} \\
\hline & Other & $\begin{array}{l}\text { Mixed; metal; zirconium } \\
\text { oxide }\end{array}$ & Metal & $5 \pm 0.5$ & Normal & $<1 \%$ & {$[26]$} \\
\hline \multirow{6}{*}{$\begin{array}{l}\Xi \\
. \Xi \\
: \circ \\
:\end{array}$} & Construction & Metal; ferroniobium & Metal & $75 \pm 22.5$ & Normal & $53 \%$ & {$[13]$} \\
\hline & Transportation & Metal; ferroniobium & Metal & $10 \pm 3$ & Normal & $53 \%$ & {$[13]$} \\
\hline & Oil and gas industry & Metal; ferroniobium & Metal & $60 \pm 18$ & Normal & $53 \%$ & {$[13]$} \\
\hline & Stainless steel & Metal; ferroniobium & Metal & $20 \pm 6$ & Normal & $53 \%$ & {$[13]$} \\
\hline & Niobium metal and alloys & Metal; ferroniobium & Metal & $20 \pm 6$ & Normal & $53 \%$ & {$[13]$} \\
\hline & Other & Mixed; niobium carbides & Metal & $15 \pm 4.5$ & Normal & $53 \%$ & {$[13]$} \\
\hline \multirow{9}{*}{ 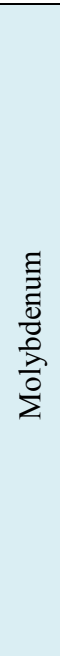 } & Industrial machinery & Metal & Metal & $25 \pm 7.5$ & Normal & $30 \%$ & [9] \\
\hline & Transportation: automotive & Metal & Metal & $14 \pm 2.8$ & Normal & $30 \%$ & {$[9]$} \\
\hline & Transportation: non-automotive & Metal & Metal & $25 \pm 10$ & Normal & $30 \%$ & {$[9]$} \\
\hline & Chemicals & $\begin{array}{l}\text { Mixed; molybdenum } \\
\text { disulfide; sodium } \\
\text { molybdate }\end{array}$ & - & & Normal & & {$[9]$} \\
\hline & Catalysts & Mixed & Molybdenum sulfide & $2 \pm 0.2$ & Normal & $30 \%$ & {$[9]$} \\
\hline & Dissipative uses & Mixed & Molybdenum sulfide & $1 \pm 3.5$ & Normal & $0 \%$ & Own assessment \\
\hline & $\begin{array}{l}\text { Pigments and flame } \\
\text { retardants }\end{array}$ & Mixed & Molybdenum sulfide & $10 \pm 3.5$ & Normal & $0 \%$ & Own assessment \\
\hline & Building and infrastructure & Metal & Metal & $50 \pm 15$ & Weibull & $30 \%$ & {$[9]$} \\
\hline & Household appliances and & Metal & Metal & $15 \pm 3$ & Weibull & $30 \%$ & {$[9]$} \\
\hline
\end{tabular}




\begin{tabular}{|c|c|c|c|c|c|c|c|}
\hline 를 & End-use & Principal form & $\begin{array}{l}\text { Form considered in } \\
\text { the analysis }\end{array}$ & $\begin{array}{l}\text { Lifetime (years): } \\
\text { average } \pm \text { standard } \\
\text { deviation }\end{array}$ & Distribution type & EOL-RR & Reference \\
\hline & electronics & & & & & & \\
\hline \multirow{4}{*}{ 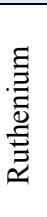 } & Electrical & Metal; ruthenium oxides & Metal & $12.5 \pm 3.5$ & Weibull & $3 \%$ & {$[27]$} \\
\hline & Electrochemical & Ruthenium oxides & Metal & $10 \pm 3.5$ & Weibull & $85 \%$ & {$[27]$} \\
\hline & Chemical & Metal; ruthenium oxides & Metal & $6.5 \pm 3.5$ & Weibull & $85 \%$ & {$[27]$} \\
\hline & Other & Metal; mixed & Metal & $15 \pm 3.5$ & Weibull & $3 \%$ & {$[27]$} \\
\hline \multirow{5}{*}{ 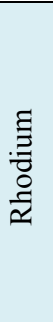 } & Autocatalyst & Metal & Metal & $16 \pm 2.57$ & Weibull & $81 \%$ & {$[27]$} \\
\hline & Glass & Metal & Metal & $2 \pm 3.5$ & Weibull & $96 \%$ & {$[27]$} \\
\hline & Chemical & $\begin{array}{l}\text { Metal; rhodium oxides; } \\
\text { rhodium chloride }\end{array}$ & Metal & $1 \pm 0.1$ & Normal & $8 \%$ & {$[27]$} \\
\hline & Electrical & Metal & Metal & $10 \pm 3.5$ & Weibull & $98 \%$ & {$[27]$} \\
\hline & Other & Metal & Metal & $15 \pm 3.5$ & Weibull & $40 \%$ & {$[27]$} \\
\hline \multirow{7}{*}{ 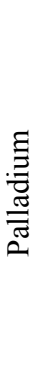 } & Autocatalyst & Metal & Metal & $16 \pm 2.57$ & Weibull & $81 \%$ & {$[27]$} \\
\hline & Electrical & Metal & Metal & $10 \pm 3.5$ & Weibull & $96 \%$ & {$[27]$} \\
\hline & Investment & Metal & Metal & $99 \pm 3.5$ & Weibull & $18 \%$ & {$[27]$} \\
\hline & Dental & Metal & Metal & $5 \pm 3.5$ & Weibull & $15 \%$ & {$[27]$} \\
\hline & Jewelry & Metal & Metal & $30 \pm 3.5$ & Weibull & $100 \%$ & {$[27]$} \\
\hline & Chemical & Metal; palladium chloride & Metal & $1 \pm 0.1$ & Normal & $100 \%$ & {$[27]$} \\
\hline & Other & Metal & Metal & $15 \pm 3.5$ & Weibull & $18 \%$ & {$[27]$} \\
\hline \multirow{6}{*}{$\stackrel{\bar{D}}{\overline{\bar{n}}}$} & Electrical and electronics & Metal & Metal & $15 \pm 2(16.9)$ & Weibull & $13 \%$ & {$[22]$} \\
\hline & Jewelry and silverware & Metal & Metal & $30 \pm 3.5(33.3)$ & Weibull & $95 \%$ & {$[22]$} \\
\hline & Coins and medals & Silver nitrate & Metal & $25 \pm 3.5(27.8)$ & Weibull & $95 \%$ & {$[22]$} \\
\hline & Photographic & Metal & Metal & $30 \pm 3.5(16.9)$ & Weibull & $50 \%$ & {$[22]$} \\
\hline & Brazing alloys and solders & Metal & Metal & $15 \pm 2(33.3)$ & Weibull & $50 \%$ & {$[22]$} \\
\hline & Other & Metal & Metal & $15 \pm 2(18.1)$ & Weibull & $50 \%$ & {$[22]$} \\
\hline
\end{tabular}




\begin{tabular}{|c|c|c|c|c|c|c|c|}
\hline $\begin{array}{l}\bar{\Xi} \\
\text { 롬 }\end{array}$ & End-use & Principal form & $\begin{array}{l}\text { Form considered in } \\
\text { the analysis }\end{array}$ & $\begin{array}{l}\text { Lifetime (years): } \\
\text { average } \pm \text { standard } \\
\text { deviation }\end{array}$ & Distribution type & EOL-RR & Reference \\
\hline \multirow{6}{*}{$\stackrel{\Xi}{\Xi}$} & Batteries (consumer) & Metal powder & Metal & $2.5 \pm 0.25$ & Weibull & $20 \%$ & {$[17]$} \\
\hline & Batteries (industrial) & Metal powder & Metal & $15 \pm 3.5$ & Weibull & $80 \%$ & {$[17]$} \\
\hline & Pigments & Cadmium sulfate & Metal $^{6}$ & $8 \pm 3.5$ & Weibull & $0 \%$ & [17] \\
\hline & Coatings & Metal & Metal & $7 \pm 3.5$ & Weibull & $0 \%$ & [17] \\
\hline & Stabilizers & Cadmium sulfate & $\mathrm{Metal}^{6}$ & $3 \pm 0.3$ & Normal & $0 \%$ & {$[17]$} \\
\hline & Other & $\begin{array}{l}\text { Mixed; Cadmium } \\
\text { stearate; Cadmium } \\
\text { laurate }\end{array}$ & $\mathrm{Metal}^{6}$ & $5 \pm 0.5$ & Normal & $0 \%$ & {$[17]$} \\
\hline \multirow{4}{*}{ 䇂 } & Thin-film coatings & Indium-tin-oxide & $\mathrm{Metal}^{7}$ & $6.5 \pm 2.76$ & Weibull & $<1 \%$ & {$[17]$} \\
\hline & Solders and alloys & Metal & Metal & $13.40 \pm 2.15$ & Weibull & $<1 \%$ & {$[17]$} \\
\hline & $\begin{array}{l}\text { Electrical components and } \\
\text { semiconductors }\end{array}$ & $\begin{array}{l}\text { Mixed; copper indium } \\
\text { gallium selenide }\end{array}$ & Metal & $13.4 \pm 2.15$ & Weibull & $<1 \%$ & {$[17]$} \\
\hline & Other & Mixed & Metal & $5 \pm 0.5$ & Normal & $<1 \%$ & {$[17]$} \\
\hline \multirow{6}{*}{$\stackrel{\Xi}{ }}$. & Solder & Metal & Metal & $13.4 \pm 2.15$ & Weibull & $0 \%$ & {$[17]$} \\
\hline & Tinplate & Metal & Metal & - & - & & {$[17]$} \\
\hline & Cans & Metal & Metal & $1 \pm 0.1$ & Normal & $0 \%$ & {$[17]$} \\
\hline & Tinning & Metal & Metal & $1 \pm 0.1$ & Normal & $0 \%$ & {$[17]$} \\
\hline & Chemicals & Mixed; organotins & $\mathrm{Metal}^{8}$ & $7 \pm 3.5$ & Weibull & & {$[17]$} \\
\hline & Stabilizer in polyvinylchloride & Organotins & $\mathrm{Metal}^{8}$ & $7 \pm 3.5$ & Weibull & $0 \%$ & {$[17]$} \\
\hline
\end{tabular}

\footnotetext{
${ }^{6}$ Cadmium sulfate is produced by melting cadmium with ammonium or sodium peroxodisulfate; it is used in electroplating and as a starting material for pigments and stabilizers, and other compound forms [28]

7 Most of production routes for indium-tin-oxide utilizes metallic indium as a starting material [29-31]

8 Tin chlorides are the starting material for butyl compounds and other organo-compounds of tin. Organotin chlorides can be formed by direct reaction of tin with unsaturated organic compounds, hydrogen halides, or organic halides [32]
} 


\begin{tabular}{|c|c|c|c|c|c|c|c|}
\hline 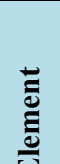 & End-use & Principal form & $\begin{array}{l}\text { Form considered in } \\
\text { the analysis }\end{array}$ & $\begin{array}{l}\text { Lifetime (years): } \\
\text { average } \pm \text { standard } \\
\text { deviation }\end{array}$ & Distribution type & EOL-RR & Reference \\
\hline & Biocide & Tributyltin & Metal $^{8}$ & $1 \pm 3.5$ & Weibull & $0 \%$ & {$[17]$} \\
\hline & Brass and bronze (construction) & Metal & Metal & $30 \pm 3.5$ & Weibull & $70 \%$ & {$[17]$} \\
\hline & Brass and bronze (vehicles) & Metal & Metal & $12 \pm 3.5$ & Weibull & $70 \%$ & {$[17]$} \\
\hline & Other & Mixed & Metal & $7 \pm 3.5$ & Weibull & $0 \%$ & {$[17]$} \\
\hline \multirow{7}{*}{ 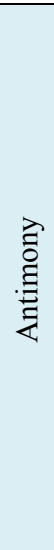 } & Flame retardants & Antimony trioxide & Metal $^{9}$ & $10 \pm 3.5$ & Weibull & $0 \%$ & {$[11]$} \\
\hline & Batteries (transportation) & Metal & Metal & $4 \pm 0.4$ & Normal & $20 \%$ & {$[11]$} \\
\hline & Batteries (industrial) & Metal & Metal & $10 \pm 3.5$ & Weibull & & {$[11]$} \\
\hline & Ceramic and glass & $\begin{array}{l}\text { Antimony trioxide; } \\
\text { sodium antimonite }\end{array}$ & Metal $^{9}$ & $10 \pm 3.5$ & Weibull & $0 \%$ & {$[11]$} \\
\hline & Chemicals & Antimony trioxide & Metal $^{9}$ & $5 \pm 0.5$ & Normal & $0 \%$ & {$[11]$} \\
\hline & Other (dissipative) & $\begin{array}{l}\text { Mixed; antimony (III) } \\
\text { sulfide; antimony (V) } \\
\text { sulfide }\end{array}$ & Metal $^{9}$ & $1 \pm 3.5$ & Weibull & $0 \%$ & Own assessment \\
\hline & Other (non dissipative) & Mixed & Metal & $5 \pm 0.5$ & Normal & & {$[11]$} \\
\hline \multirow{5}{*}{$\stackrel{\Xi}{\Xi}$} & Solar and photovoltaic market & Cadmium telluride & Metal $^{10}$ & $30 \pm 0.01$ & Normal & $27 \%$ & {$[8]$} \\
\hline & Thermoelectric devices & Metal & Metal & $10 \pm 0.01$ & Normal & $27 \%$ & {$[8]$} \\
\hline & $\begin{array}{l}\text { Metallurgy (iron and steel } \\
\text { products) }\end{array}$ & Metal & Metal & $30 \pm 3.5$ & Weibull & $0 \%$ & {$[8]$} \\
\hline & $\begin{array}{c}\text { Metallurgy (additives to } \\
\text { nonferrous alloys) }\end{array}$ & Metal & Metal & $20 \pm 3.5$ & Weibull & & {$[8]$} \\
\hline & Rubber applications & $\begin{array}{l}\text { Cadmium sulfotelluride; } \\
\text { cadmium telluride; }\end{array}$ & Metal $^{10}$ & $10 \pm 3.5$ & Weibull & $0 \%$ & {$[8]$} \\
\hline
\end{tabular}

9 Antimony trioxide can be obtained by roasting antimony trisulfide or by its hydrolysis with superheated steam, by combustion of antimony in presence of oxygen, and by alkaline hydrolysis of antimony halides [33]. Antimony trisulfide is prepared from the elements at temperature 500-900 Celsius degrees. [34]

10 Cadmium telluride can be produced from elemental cadmium and tellurium. [35, 36] 


\begin{tabular}{|c|c|c|c|c|c|c|c|}
\hline 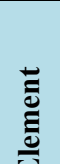 & End-use & Principal form & $\begin{array}{l}\text { Form considered in } \\
\text { the analysis }\end{array}$ & $\begin{array}{l}\text { Lifetime (years): } \\
\text { average } \pm \text { standard } \\
\text { deviation }\end{array}$ & Distribution type & EOL-RR & Reference \\
\hline & & $\begin{array}{l}\text { cerium tellurium } \\
\text { molybdate }\end{array}$ & & & & & \\
\hline & $\begin{array}{l}\text { Chemicals and catalysts (non } \\
\text { dissipative) }\end{array}$ & $\begin{array}{l}\text { Organic tellurides; } \\
\text { sodium tellurite }\end{array}$ & Metal $^{10}$ & $5 \pm 3.5$ & Weibull & $0 \%$ & {$[8]$} \\
\hline & $\begin{array}{l}\text { Chemicals and catalysts } \\
\text { (dissipative) }\end{array}$ & $\begin{array}{l}\text { Organic tellurides; } \\
\text { sodium tellurite }\end{array}$ & Metal $^{10}$ & $1 \pm 3.5$ & Weibull & & [8] \\
\hline \multirow{7}{*}{ 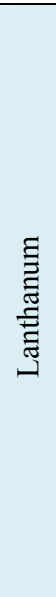 } & Fluid catalysts & $\begin{array}{l}\text { Mixed; lanthanum } \\
\text { oxides; lanthanum } \\
\text { chlorides }\end{array}$ & Lanthanum oxide & $5 \pm 2$ & Normal & $0 \%$ & {$[25]$} \\
\hline & Battery alloys & Lanthanum oxide & Lanthanum oxide & $5 \pm 2$ & Normal & $0 \%$ & {$[25]$} \\
\hline & Glass polishing & Lanthanum oxide & Lanthanum oxide & $1 \pm 0.1$ & Normal & $0 \%$ & {$[25]$} \\
\hline & Metallurgy (excluding batteries) & Mischmetal; La oxides & Lanthanum oxide & $30 \pm 10$ & Normal & $0 \%$ & {$[25]$} \\
\hline & Glass additives & $\begin{array}{l}\text { Lanthanum oxides; } \\
\text { lanthanum (III) fluoride }\end{array}$ & Lanthanum oxide & $5 \pm 1$ & Normal & $0 \%$ & {$[25]$} \\
\hline & Phosphors & Lanthanum oxide & Lanthanum oxide & $1 \pm 0.5$ & Normal & $0 \%$ & {$[25]$} \\
\hline & Other & $\begin{array}{l}\text { Mixed; mischmetal; } \\
\text { lanthanum (III) fluoride }\end{array}$ & Lanthanum oxide & $5 \pm 1$ & Normal & $0 \%$ & {$[25]$} \\
\hline \multirow{6}{*}{ 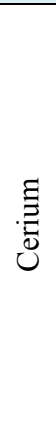 } & Glass polishing & Cerium oxide & Cerium oxide & $1 \pm 0.1$ & Normal & $0 \%$ & {$[25]$} \\
\hline & Glass additives & Cerium oxide & Cerium oxide & $5 \pm 1$ & Normal & $0 \%$ & {$[25]$} \\
\hline & Autocatalytic converters & Cerium oxide & Cerium oxide & $10 \pm 5$ & Normal & $0 \%$ & {$[25]$} \\
\hline & Metallurgy (excluding batteries) & Mixed; cerium oxide & Cerium oxide & $30 \pm 10$ & Normal & $0 \%$ & {$[25]$} \\
\hline & Battery alloys & $\begin{array}{l}\text { Mischmetal; cerium } \\
\text { oxide } \\
\text { (Cerium oxide) }\end{array}$ & Cerium oxide & $5 \pm 2$ & Normal & $0 \%$ & {$[25]$} \\
\hline & Phosphors & Cerium oxide & Cerium oxide & $1 \pm 0.5$ & Normal & $0 \%$ & {$[25]$} \\
\hline
\end{tabular}




\begin{tabular}{|c|c|c|c|c|c|c|c|}
\hline$\stackrel{\bar{E}}{E}$ & End-use & Principal form & $\begin{array}{l}\text { Form considered in } \\
\text { the analysis }\end{array}$ & $\begin{array}{l}\text { Lifetime (years): } \\
\text { average } \pm \text { standard } \\
\text { deviation }\end{array}$ & Distribution type & EOL-RR & Reference \\
\hline & Other & $\begin{array}{l}\text { Mixed; mischmetal; } \\
\text { cerium sulfide }\end{array}$ & Cerium oxide & $5 \pm 1$ & Normal & $0 \%$ & {$[25]$} \\
\hline \multirow{6}{*}{ 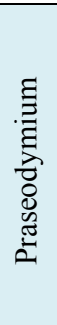 } & Neodymium magnets & Praseodymium oxide & & $20 \pm 5$ & Normal & $0 \%$ & {$[25]$} \\
\hline & Glass polishing & Praseodymium oxide & Praseodymium oxide & $1 \pm 0.1$ & Normal & $0 \%$ & {$[25]$} \\
\hline & Metallurgy (exc. batteries) & Mixed; metal & Praseodymium oxide & $30 \pm 10$ & Normal & $0 \%$ & {$[25]$} \\
\hline & Battery alloys & Praseodymium oxide & Praseodymium oxide & $5 \pm 2$ & Normal & $0 \%$ & {$[25]$} \\
\hline & Autocatalytic converters & Praseodymium oxide & Praseodymium oxide & $10 \pm 5$ & Normal & $0 \%$ & {$[25]$} \\
\hline & Other & Mixed; mischmetal & Praseodymium oxide & $1 \pm 0.5$ & Normal & $0 \%$ & {$[25]$} \\
\hline \multirow{6}{*}{ 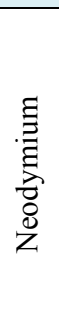 } & Neodymium magnets & Neodymium iron boron & Neodymium oxide & $20 \pm 5$ & Normal & $0 \%$ & {$[25]$} \\
\hline & Metallurgy (excluding batteries) & Neodymium oxide & Neodymium oxide & $30 \pm 10$ & Normal & $0 \%$ & {$[25]$} \\
\hline & Battery alloys & Neodymium oxide & Neodymium oxide & $5 \pm 2$ & Normal & $0 \%$ & {$[25]$} \\
\hline & Ceramics & Neodymium oxide & Neodymium oxide & $5 \pm 2$ & Normal & $0 \%$ & {$[25]$} \\
\hline & Autocatalytic converters & Neodymium oxide & Neodymium oxide & $10 \pm 5$ & Normal & $0 \%$ & {$[25]$} \\
\hline & Other & Neodymium oxide & Neodymium oxide & $1 \pm 0.5$ & Normal & $0 \%$ & {$[25]$} \\
\hline \multirow{2}{*}{ 急 } & Battery alloys & $\begin{array}{l}\text { Mischmetal; samarium } \\
\text { oxide }\end{array}$ & Samarium oxide & $5 \pm 2$ & Normal & $0 \%$ & {$[25]$} \\
\hline & Other & $\begin{array}{l}\text { Mixed; samarium iodide; } \\
\text { samarium-cobalt alloys }\end{array}$ & Samarium oxide & $1 \pm 0.1$ & Normal & $0 \%$ & {$[25]$} \\
\hline 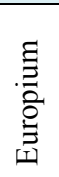 & Phosphors & Europium oxide & Europium oxide & $4 \pm 1$ & Normal & $0 \%$ & {$[25]$} \\
\hline \multirow{3}{*}{ 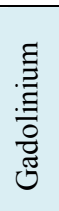 } & Neodymium magnets & $\begin{array}{l}\text { Gadolinium gallium } \\
\text { alloys }\end{array}$ & Gadolinium oxide & $20 \pm 5$ & Normal & $0 \%$ & {$[25]$} \\
\hline & Phosphors & Gadolinium oxide & Gadolinium oxide & $4 \pm 1$ & Normal & $0 \%$ & {$[25]$} \\
\hline & Medical imaging & Gadolinium compounds & Gadolinium oxide & $1 \pm 0.1$ & Normal & $0 \%$ & {$[25]$} \\
\hline
\end{tabular}




\begin{tabular}{|c|c|c|c|c|c|c|c|}
\hline$\underset{\vec{E}}{\vec{\Xi}}$ & End-use & Principal form & $\begin{array}{l}\text { Form considered in } \\
\text { the analysis }\end{array}$ & $\begin{array}{l}\text { Lifetime (years): } \\
\text { average } \pm \text { standard } \\
\text { deviation }\end{array}$ & Distribution type & EOL-RR & Reference \\
\hline \multirow{2}{*}{ 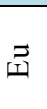 } & Phosphors & Terbium oxide & Terbium oxide & $4 \pm 2$ & Normal & $0 \%$ & {$[25]$} \\
\hline & Neodymium magnets & Mixed & Terbium oxide & $20 \pm 5$ & Normal & $0 \%$ & {$[25]$} \\
\hline 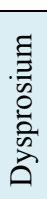 & Neodymium magnets & Mixed & Dysprosium oxide & $20 \pm 5$ & Normal & $0 \%$ & {$[25]$} \\
\hline \multirow{6}{*}{ 刍 } & Superalloys: aerospace & Metal & Metal & $5 \pm 0.5$ & Normal & $<1 \%$ & {$[26]$} \\
\hline & Nuclear energy control rods & Metal & Metal & $20 \pm 3.5$ & Normal & $<1 \%$ & {$[26]$} \\
\hline & $\begin{array}{l}\text { Superalloys: fuel reprocessing } \\
\text { plants }\end{array}$ & Metal & Metal & $16 \pm 1.91$ & Weibull & $<1 \%$ & {$[26]$} \\
\hline & Refractory & Hafnium oxide & Metal & $2 \pm 0.2$ & Normal & $<1 \%$ & {$[26]$} \\
\hline & $\begin{array}{l}\text { Manufacturing: plasma cutting } \\
\text { tools }\end{array}$ & Hafnium carbide & Metal $^{11}$ & $17.3 \pm 1.97$ & Weibull & $<1 \%$ & {$[26]$} \\
\hline & Other & Mixed & Metal & $5 \pm 0.5$ & Normal & $<1 \%$ & {$[26]$} \\
\hline \multirow{4}{*}{ 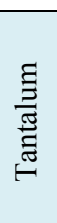 } & Electronic components & Metal & Metal & $13.4 \pm 2.15$ & Weibull & $0 \%$ & {$[9]$} \\
\hline & Machinery & Metal; metal carbide & Metal & $1 \pm 0.1$ & Normal & $50 \%$ & [9] \\
\hline & Transportation & Metal & Metal & $5 \pm 0.5$ & Normal & $70 \%$ & {$[9]$} \\
\hline & Other & Mixed & Metal & $5 \pm 0.5$ & Normal & $0 \%$ & {$[9]$} \\
\hline \multirow{3}{*}{ 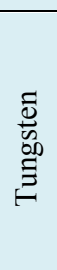 } & $\begin{array}{l}\text { Cemented carbides (cutting } \\
\text { tools) }\end{array}$ & Metal carbide & Metal $^{12}$ & $1 \pm 0.1$ & Normal & $50 \%$ & {$[9]$} \\
\hline & Cemented carbides (dies) & Metal carbide & Metal $^{12}$ & $1 \pm 0.1$ & Normal & $75 \%$ & [9] \\
\hline & $\begin{array}{l}\text { Cemented carbides (mining and } \\
\text { construction tools) }\end{array}$ & Metal carbide & Metal $^{12}$ & $1 \pm 0.1$ & Normal & $15 \%$ & {$[9]$} \\
\hline
\end{tabular}

\footnotetext{
${ }^{11}$ Hafnium carbide is obtained by carbothermic reduction of hafnia (hafnium oxide) in an induction heated graphite lined vacuum furnace. [24]

12 Direct carburization of tungsten oxide (reduced by carbon) is carried out to produce tungsten carbide. [38]
} 


\begin{tabular}{|c|c|c|c|c|c|c|c|}
\hline \multirow{7}{*}{ 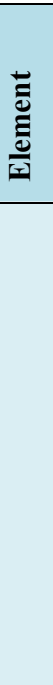 } & End-use & Principal form & $\begin{array}{l}\text { Form considered in } \\
\text { the analysis }\end{array}$ & $\begin{array}{l}\text { Lifetime (years): } \\
\text { average } \pm \text { standard } \\
\text { deviation }\end{array}$ & Distribution type & EOL-RR & Reference \\
\hline & Mill products & Metal & Metal & $5 \pm 0.5$ & Normal & $0 \%$ & [9] \\
\hline & Steel & Metal & Metal & $1 \pm 0.1$ & Normal & $50 \%$ & [9] \\
\hline & Superalloys & Metal & Metal & $5 \pm 0.5$ & Normal & $80 \%$ & [9] \\
\hline & Other & $\begin{array}{l}\text { Mixed; ammonium } \\
\text { paratungstate; tungsten } \\
\text { oxide; metal powder }\end{array}$ & Metal & - & - & - & \\
\hline & Other (catalysts) & Metal & Metal & $5 \pm 0.5$ & Normal & $50 \%$ & $\begin{array}{l}\text { Own assessment } \\
\text { based on [9] }\end{array}$ \\
\hline & Other (miscellaneous) & Metal & Metal & $5 \pm 0.5$ & Normal & $0 \%$ & [9] \\
\hline \multirow{3}{*}{.尹ٍ } & Superalloys & Metal & Metal & $5 \pm 0.5$ & Normal & $55 \%$ & [9] \\
\hline & Catalysts & Metal & Metal & $5 \pm 0.5$ & Normal & $90 \%$ & {$[9]$} \\
\hline & Other & Platinum-rhenium alloys & Metal & $5 \pm 0.5$ & Normal & $0 \%$ & [9] \\
\hline \multirow{4}{*}{$\stackrel{\Xi}{\Xi}$} & Electrical & Metal & Metal & $2 \pm 3.5$ & Weibull & $45 \%$ & {$[27]$} \\
\hline & Electrochemical & Iridium oxide & Metal & $6.5 \pm 3.5$ & Weibull & $8 \%$ & {$[27]$} \\
\hline & Chemical & Metal & Metal & $2.5 \pm 3.5$ & Weibull & $45 \%$ & {$[27]$} \\
\hline & Other & Metal & Metal & $15 \pm 3.5$ & Weibull & $8 \%$ & {$[27]$} \\
\hline \multirow{8}{*}{$\stackrel{\Xi}{\stackrel{\Xi}{\Xi}}$} & Autocatalyst & Metal & Metal & $16 \pm 2.57$ & Weibull & $80 \%$ & {$[27]$} \\
\hline & Jewelry & Metal & Metal & $30 \pm 3.5$ & Weibull & $98 \%$ & {$[27]$} \\
\hline & Investment & Metal & Metal & $999 \pm 3.5$ & Weibull & $99 \%$ & {$[27]$} \\
\hline & Chemical & $\begin{array}{l}\text { Metal; powder; contact } \\
\text { gauzes; platinum oxide }\end{array}$ & Metal & $1 \pm 0.1$ & Weibull & $80 \%$ & {$[27]$} \\
\hline & Glass & Metal & Metal & $2 \pm 3.5$ & Weibull & $99 \%$ & {$[27]$} \\
\hline & Electrical & Metal & Metal & $10 \pm 3.5$ & Weibull & $97 \%$ & [27] \\
\hline & Medical and biomedical & Metal compounds & Metal & $8 \pm 3.5$ & Weibull & $15 \%$ & {$[27]$} \\
\hline & Petroleum & Metal & Metal & $5 \pm 3.5$ & Weibull & $98 \%$ & {$[27]$} \\
\hline
\end{tabular}




\begin{tabular}{|c|c|c|c|c|c|c|c|}
\hline 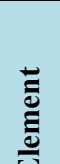 & End-use & Principal form & $\begin{array}{l}\text { Form considered in } \\
\text { the analysis }\end{array}$ & \begin{tabular}{|l|} 
Lifetime (years): \\
average \pm standard \\
deviation
\end{tabular} & Distribution type & EOL-RR & Reference \\
\hline & Other & Metal & Metal & $15 \pm 3.5$ & Weibull & $15 \%$ & {$[27]$} \\
\hline \multirow{5}{*}{$\frac{7}{0}$} & Jewelry & Metal & Metal & $40 \pm 3.5(44.5)$ & Weibull & $95 \%$ & {$[22]$} \\
\hline & Investment & Metal & Metal & $99 \pm 3.5$ & Weibull & $99 \%$ & {$[27]$} \\
\hline & Electronics & Metal & Metal & $13.4 \pm 2.15(15.1)$ & Weibull & $13 \%$ & {$[22]$} \\
\hline & Dental and medical & Metal & Metal & $37 \pm 1.6(41.3)$ & Weibull & $18 \%$ & {$[22]$} \\
\hline & Other & Metal & Metal & $13.1 \pm 1.79(15.6)$ & Weibull & $80 \%$ & {$[22]$} \\
\hline \multirow{9}{*}{ 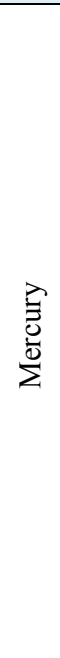 } & $\begin{array}{l}\text { Artisanal and small-scale } \\
\text { mining }\end{array}$ & Amalgam & Metal & $1 \pm 0.1$ & Normal & $0 \%$ & {$[11]$} \\
\hline & $\begin{array}{l}\text { Vinyl chloride monomer } \\
\text { production }\end{array}$ & Metal; mercury chloride & Metal & $1 \pm 0.1$ & Normal & $43 \%$ & {$[11]$} \\
\hline & $\begin{array}{c}\text { Chlorine caustic soda } \\
\text { manufacturing }\end{array}$ & Metal; mercury chloride & Metal & $1 \pm 0.1$ & Normal & $16 \%$ & {$[11]$} \\
\hline & Electrical (batteries) & Metal; mercury oxide & Metal & $2 \pm 0.2$ & Normal & $15 \%$ & {$[11]$} \\
\hline & Dental equipment and supplies & Amalgam & Metal & $7 \pm 3.5$ & Weibull & $15 \%$ & {$[11]$} \\
\hline & Electrical and electronic devices & Metal & Metal & $13.4 \pm 2.15$ & Weibull & $15 \%$ & {$[11]$} \\
\hline & Measuring and control devices & Metal & Metal & $20 \pm 3.5$ & Weibull & $15 \%$ & {$[11]$} \\
\hline & Electrical lighting & Metal & Metal & $3 \pm 0.3$ & Normal & $15 \%$ & {$[11]$} \\
\hline & Other & Mixed; mercury sulfide & Metal & $5 \pm 0.5$ & Normal & $0 \%$ & {$[11]$} \\
\hline \multirow{3}{*}{$\stackrel{\Xi}{\Xi}$} & Electronics & $\begin{array}{l}\text { Metal; thallium arsenic } \\
\text { selenium }\end{array}$ & Metal & $13.4 \pm 2.15$ & Weibull & $<1 \%$ & {$[11]$} \\
\hline & Pesticides & $\begin{array}{l}\text { Mixed; thallium (III) } \\
\text { sulfate }\end{array}$ & Metal & $2 \pm 0.2$ & Normal & $<1 \%$ & {$[11]$} \\
\hline & Other & Mixed; thallium-201 & Metal & $5 \pm 0.5$ & Normal & $<1 \%$ & {$[11]$} \\
\hline \multirow{2}{*}{ 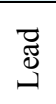 } & Batteries (transportation) & Batteries (transportation) & Metal & $4 \pm 0.4$ & Normal & $75 \%$ & {$[17]$} \\
\hline & Batteries (industrial) & Batteries (industrial) & Metal & $10 \pm 3.5$ & Weibull & $75 \%$ & {$[17]$} \\
\hline
\end{tabular}




\begin{tabular}{|c|c|c|c|c|c|c|c|}
\hline Е & End-use & Principal form & $\begin{array}{l}\text { Form considered in } \\
\text { the analysis }\end{array}$ & $\begin{array}{l}\text { Lifetime (years): } \\
\text { average } \pm \text { standard } \\
\text { deviation }\end{array}$ & Distribution type & EOL-RR & Reference \\
\hline & Cable sheathing & Cable sheathing & Metal & $15.5 \pm 2.67$ & Weibull & $30 \%$ & {$[17]$} \\
\hline & Semi-manufactured and alloys & $\begin{array}{l}\text { Semi-manufactured and } \\
\text { alloys }\end{array}$ & Metal & $13.50 \pm 1.81$ & Weibull & $60 \%$ & [17] \\
\hline & Chemicals & Chemicals & Metal $^{13}$ & $1 \pm 0.1$ & Normal & $0 \%$ & {$[17]$} \\
\hline & Other & Other & Metal $^{13}$ & $13.5 \pm 1.81$ & Weibull & $0 \%$ & {$[17]$} \\
\hline \multirow{4}{*}{ 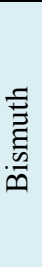 } & $\begin{array}{l}\text { Fusible alloys, solders, and } \\
\text { ammunition }\end{array}$ & Metal & Metal & $13.4 \pm 2.15$ & Weibull & $<1 \%$ & {$[11]$} \\
\hline & Metallurgical additives & Metal & Metal & $13.5 \pm 1.81$ & Weibull & $<1 \%$ & [11] \\
\hline & Pharmaceutical and chemicals & Mixed; bismuth salicylate & Metal & $5 \pm 0.5$ & Normal & $<1 \%$ & [11] \\
\hline & Other & Mixed & Metal & $5 \pm 0.5$ & Normal & $<1 \%$ & {$[11]$} \\
\hline \multirow{4}{*}{ 导 } & Welding electrodes & Copper thorium alloys & Thorium oxide & $1 \pm 0.1$ & Normal & $0 \%$ & {$[26]$} \\
\hline & Lighting & Thorium oxide & Thorium oxide & $1 \pm 0.1$ & Normal & $0 \%$ & {$[26]$} \\
\hline & Refractory & Thorium oxide & Thorium oxide & $2 \pm 0.2$ & Normal & $0 \%$ & {$[26]$} \\
\hline & Other & Mixed & Thorium oxide & $5 \pm 0.5$ & Normal & $0 \%$ & {$[26]$} \\
\hline \multirow{2}{*}{$\stackrel{\Xi}{\Xi}$} & Nuclear energy & $\begin{array}{l}\text { Uranium oxide; uranium- } \\
\text { 235; uranium-238 }\end{array}$ & Uranium oxide & $1 \pm 0.1$ & Normal & $3 \%$ & [26] \\
\hline & Naval use & $\begin{array}{l}\text { Uranium oxide; uranium- } \\
235 \text {; uranium- } 238\end{array}$ & Uranium oxide & $10 \pm 3.5$ & Normal & $0 \%$ & {$[26]$} \\
\hline
\end{tabular}

${ }^{13}$ Lead compounds are commonly produced from metal lead through a series of processes that involve the direct or indirect preparation of lead monoxide. [39] 
2 values for each metal form considered in the analysis. Modified from Nuss and Eckelman (2014). ${ }^{2}$

\begin{tabular}{|c|c|c|c|c|c|c|c|}
\hline Element & Form & $\begin{array}{c}\text { GER } \\
{[\mathrm{MJ} / \mathbf{k g}]}\end{array}$ & $\begin{array}{c}\text { GER - 2.5\% } \\
\text { [MJ/kg] }\end{array}$ & $\begin{array}{c}\text { GER - 97.5\% } \\
\text { [MJ } / \mathrm{kg}]\end{array}$ & $\begin{array}{c}\text { GWP 100a } \\
\text { [kg CO2-eq/ } \\
\text { kg] }\end{array}$ & $\begin{array}{c}\text { GWP }-2.5 \%[\mathrm{~kg} \\
\mathrm{CO} 2-\mathrm{eq} / \mathrm{kg}]\end{array}$ & $\begin{array}{c}\text { GWP - 97.5\% [kg } \\
\text { CO2-eq/ kg] }\end{array}$ \\
\hline $\mathrm{Al}$ & Metal & 194 & 158 & 246 & 12.2 & 10.3 & 14.5 \\
\hline $\mathrm{Sc}$ & $\mathrm{Sc}_{2} \mathrm{O}_{3}$ & 97200 & 53800 & 259000 & 5710 & 5290 & 6570 \\
\hline \multirow{2}{*}{$\mathrm{Ti}$} & $\mathrm{TiO} 2$ (from Ilmenite) & 80.7 & 67.6 & 95.2 & 7.2 & 6.6 & 7.8 \\
\hline & Metal & 703 & 503 & 956 & 45.1 & 32 & 61.3 \\
\hline V & Metal & 516 & 306 & 844 & 33.1 & 19.4 & 53 \\
\hline \multirow{2}{*}{$\mathrm{Cr}$} & $\mathrm{FeCr}$ & 37.3 & 31.6 & 45.0 & 1.9 & 1.6 & 2.3 \\
\hline & $\mathrm{Na}_{2} \mathrm{Cr}_{2} \mathrm{O}_{7}$ & 54.6 & 43.2 & 72.0 & 4.8 & 4.4 & 5.3 \\
\hline \multirow{2}{*}{$\mathrm{Mn}$} & FeMn & 23.5 & 18.9 & 29.4 & 1.0 & 0.8 & 1.2 \\
\hline & $\mathrm{Mn}_{2} \mathrm{O}_{3}$ & 37.9 & 26.4 & 51.4 & 2.4 & 1.9 & 3.1 \\
\hline $\mathrm{Fe}$ & Metal & 23.1 & 20.9 & 25.5 & 1.5 & 1.4 & 1.7 \\
\hline Co & Metal & 128 & 93.2 & 179 & 8.3 & 6 & 11.5 \\
\hline $\mathrm{Ni}$ & $\mathrm{FeNi}$ & 156 & 135 & 183 & 9.2 & 8.4 & 10.2 \\
\hline $\mathrm{Cu}$ & Metal & 59.3 & 49.7 & 70.8 & 3.1 & 2.6 & 3.7 \\
\hline $\mathrm{Zn}$ & Metal & 54.4 & 37.2 & 82.1 & 3.2 & 2.5 & 4.4 \\
\hline $\mathrm{Ga}$ & Semiconductor grade & 3030 & 1520 & 5350 & 205 & 128 & 331 \\
\hline $\mathrm{Ge}$ & Metal & 2890 & 1870 & 4570 & 170 & 121 & 252 \\
\hline \multirow{2}{*}{ As } & $\mathrm{As}_{2} \mathrm{O}_{3}$ & 3.1 & 2.6 & 3.8 & 0.2 & 0.1 & 0.2 \\
\hline & Metal & 16.5 & 14.0 & 19.5 & 1.3 & 1.1 & 1.6 \\
\hline $\mathrm{Se}$ & $\mathrm{Se}$ & 65.5 & 49.2 & 88.4 & 3.6 & 2.7 & 4.8 \\
\hline $\mathrm{Y}$ & $\mathrm{Y}_{2} \mathrm{O}_{3}$ & 295 & 231 & 374 & 15.1 & 11.9 & 19.2 \\
\hline \multirow{2}{*}{$\mathrm{Zr}$} & $\mathrm{ZrO}_{2}$ & 62.2 & 47.4 & 82.4 & 3.4 & 2.7 & 4.2 \\
\hline & Pure metal & 576 & 298 & 1140 & 21.4 & 11.7 & 37.8 \\
\hline
\end{tabular}




\begin{tabular}{|c|c|c|c|c|c|c|c|}
\hline Element & Form & $\begin{array}{c}\text { GER } \\
{[\mathrm{MJ} / \mathrm{kg}]}\end{array}$ & $\begin{array}{c}\text { GER - 2.5\% } \\
{[\mathrm{MJ} / \mathrm{kg}]}\end{array}$ & $\begin{array}{c}\text { GER }-97.5 \% \\
{[\mathrm{MJ} / \mathrm{kg}]}\end{array}$ & $\begin{array}{c}\text { GWP 100a } \\
{\left[\begin{array}{c}\text { [kg CO2-eq/ } \\
\text { kg] }\end{array}\right.}\end{array}$ & $\begin{array}{c}\text { GWP }-2.5 \%[\mathrm{~kg} \\
\text { CO2-eq/ kg] }\end{array}$ & $\begin{array}{c}\text { GWP }-97.5 \%[\mathrm{~kg} \\
\text { CO2-eq/ kg] }\end{array}$ \\
\hline \multirow{2}{*}{$\mathrm{Nb}$} & Pure metal & 639 & 453 & 902 & 46.6 & 34.4 & 64 \\
\hline & $\mathrm{FeNb}$ & 114 & 82.3 & 153 & 8.3 & 6.2 & 11.1 \\
\hline \multirow{2}{*}{ Mo } & Pure metal & 235 & 115 & 455 & 11.7 & 4.8 & 20 \\
\hline & $\mathrm{MoS}_{2}$ (from copper ores) & 150 & 122 & 187 & 7.3 & 5.7 & 9.3 \\
\hline $\mathrm{Ru}$ & $\begin{array}{l}\text { Metal (from Bushveld Mine, PGM- } \\
\text { dominated) }\end{array}$ & 40700 & 29200 & 54800 & 2060 & 1510 & 2700 \\
\hline $\mathrm{Pd}$ & $\mathrm{Pd}(\mathrm{RU})$ & 92200 & 68200 & 118000 & 5110 & 4090 & 6450 \\
\hline $\mathrm{Ag}$ & Metal (from gold ores) & 6740 & 5430 & 8660 & 405 & 313 & 516 \\
\hline $\mathrm{Cd}$ & Metal & 27.5 & 20.6 & 36.1 & 1.4 & 1.1 & 1.9 \\
\hline In & Metal & 1720 & 1170 & 2520 & 102 & 78.8 & 155 \\
\hline $\mathrm{Sn}$ & Metal & 321 & 201 & 496 & 17.1 & 11 & 26.8 \\
\hline $\mathrm{Sb}$ & Metal (from stibnite) & 141 & 68.4 & 342 & 12.9 & 7.2 & 23.9 \\
\hline $\mathrm{Te}$ & Metal & 435 & 332 & 559 & 21.9 & 17.1 & 27.3 \\
\hline $\mathrm{La}$ & $\mathrm{La}_{2} \mathrm{O}_{3}$ (from bastnaesite, China) & 216 & 172 & 275 & 11.0 & 8.8 & 14.3 \\
\hline $\mathrm{Ce}$ & $\mathrm{CeO}_{2}$ (from bastnaesite, China) & 252 & 198 & 326 & 12.9 & 10.1 & 16.4 \\
\hline $\operatorname{Pr}$ & $\operatorname{Pr}_{6} \mathrm{O}_{11}$ (from bastnaesite, China) & 376 & 301 & 475 & 19.2 & 14.9 & 24.7 \\
\hline $\mathrm{Nd}$ & $\mathrm{Nd}_{2} \mathrm{O}_{3}$ (from bastnaesite, China) & 344 & 267 & 437 & 17.6 & 13.8 & 22.9 \\
\hline $\mathrm{Sm}$ & $\mathrm{Sm}_{2} \mathrm{O}_{3}$ (from bastnaesite, China) & 1160 & 915 & 1490 & 59.1 & 46.1 & 77.2 \\
\hline $\mathrm{Eu}$ & $\mathrm{Eu}_{2} \mathrm{O}_{3}$ (from bastnaesite, China) & 7750 & 6020 & 10100 & 396 & 314 & 505 \\
\hline $\mathrm{Gd}$ & $\mathrm{Gd}_{2} \mathrm{O}_{3}$ (from bastnaesite, China) & 914 & 726 & 1170 & 46.6 & 37.2 & 60.7 \\
\hline $\mathrm{Tb}$ & $\mathrm{Tb}_{4} \mathrm{O}_{7}$ (from bastnaesite, China) & 5820 & 4600 & 7510 & 297 & 237 & 385 \\
\hline Dy & $\mathrm{Dy}_{2} \mathrm{O}_{3}$ (from bastnaesite, China) & 1170 & 904 & 1480 & 59.6 & 46.9 & 76.4 \\
\hline Hf & Metal & 4220 & 2200 & 8520 & 157 & 85.5 & 276 \\
\hline $\mathrm{Ta}$ & Metal & 4360 & 3460 & 5570 & 260 & 206 & 331 \\
\hline $\mathrm{W}$ & W (ProBas) & 52.4 & 44.9 & 61.5 & 3.9 & 3.2 & 4.7 \\
\hline
\end{tabular}




\begin{tabular}{|c|c|c|c|c|c|c|c|}
\hline Element & Form & $\begin{array}{c}\text { GER } \\
{[\mathrm{MJ} / \mathrm{kg}]}\end{array}$ & $\begin{array}{c}\text { GER - 2.5\% } \\
\text { [MJ/kg] }\end{array}$ & $\begin{array}{c}\text { GER }-97.5 \% \\
{[\mathrm{MJ} / \mathrm{kg}]}\end{array}$ & $\begin{array}{c}\text { GWP 100a } \\
\text { [kg CO2-eq/ } \\
\text { kg] }\end{array}$ & $\begin{array}{c}\text { GWP }-2.5 \%[\mathrm{~kg} \\
\text { CO2-eq/ kg] }\end{array}$ & $\begin{array}{c}\text { GWP }-97.5 \%[\mathrm{~kg} \\
\text { CO2-eq/ kg] }\end{array}$ \\
\hline & W (Idemat) & 215 & 152 & 286 & 21.2 & 15.3 & 29.4 \\
\hline $\operatorname{Re}$ & Metal (from molybdenite ore) & 9040 & 4470 & 16300 & 450 & 213 & 836 \\
\hline $\mathrm{Ir}$ & Metal & 175000 & 130000 & 224000 & 9670 & 7580 & 12600 \\
\hline $\mathrm{Pt}$ & $\begin{array}{l}\text { Metal (from Bushveld Mine, PGM- } \\
\text { dominated) }\end{array}$ & 308000 & 219000 & 414000 & 15600 & 11700 & 21000 \\
\hline $\mathrm{Au}$ & Metal (from gold ores) & 245000 & 199000 & 315000 & 13600 & 11100 & 16800 \\
\hline $\mathrm{Hg}$ & Metal & 179 & 110 & 280 & 12.1 & 7.7 & 18.6 \\
\hline $\mathrm{Tl}$ & Metal & 5160 & 4220 & 6180 & 376 & 189 & 681 \\
\hline $\mathrm{Pb}$ & Metal (from lead/zinc ores) & 27.9 & 22.6 & 35.5 & 2.4 & 2 & 2.8 \\
\hline $\mathrm{Bi}$ & Metal (from lead/zinc ores) & 697 & 332 & 2320 & 58.9 & 53.7 & 66.5 \\
\hline $\mathrm{Th}$ & $\mathrm{ThO}_{2}$ & 1260 & 960 & 1670 & 74.9 & 62.4 & 91.8 \\
\hline $\mathrm{U}$ & $\mathrm{UO}_{2}$ & 1270 & 826 & 2000 & 90.7 & 61.3 & 140 \\
\hline
\end{tabular}


1 Table S6. Uncertainty ranges applied for sensitivity analysis, where GWP is global warming 2 potential.

$\begin{array}{lc}\text { Paramater } & \text { Uncertainty range } \\ \text { Flows into use } & \pm 10 \% \\ \text { In-use dissipation rates } & \pm 20 \% \\ \text { End-of-life recycling rates } & \pm 10 \% \\ \text { GWP (lower percentile) } & 2.5 \% \\ \text { GWP (upper percentile) } & 97.5 \%\end{array}$

3 
1 Figure S1. Uncertainty ranges (in grey) of the results for each use evaluated.
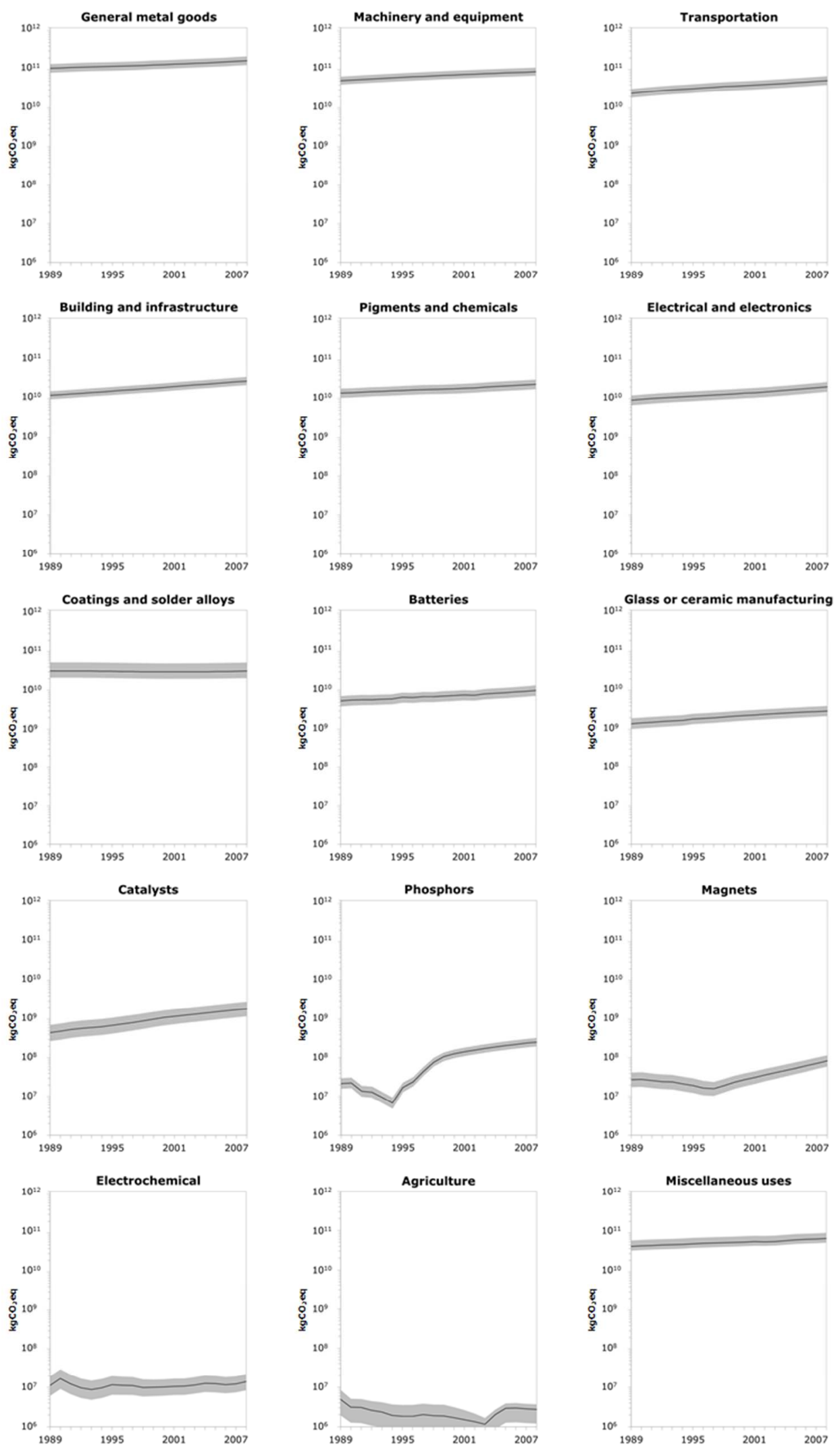


\section{References}

2 [1] Nuss, P.; Eckelman, M. J. Life cycle assessment of metals: a scientific synthesis. PloS One 2014, 39 , e101298.

4 [2] UNEP, 2013. Environmental Risks and Challenges of Anthropogenic Metals Flows and Cycles, 5 A Report of the Working Group on the Global Metal Flows to the International Resource Panel. van der Voet, E.; Salminen, R.; Eckelman, M.; Mudd, G.; Norgate, T.; Hischier, R.

7 [3] Liu, G.; Bangs, C. E.; Muller, D. B. Stock dynamics and emission pathways of the global

[4] Wang, T.; Müller, D. B.; Graedel, T. E. Forging the anthropogenic iron cycle. Environ. Sci. Technol. 2007, 41, 5120-5129.

Glöser, S.; Soulier, M.; Tercero Espinoza, L. A. Dynamic analysis of global copper flows. Global stocks, postconsumer material flows, recycling indicators, and uncertainty evaluation. Environ. Sci. Technol. 2013, 47, 6564-6572.

Kavlak, G.; Graedel, T. Global anthropogenic selenium cycles for 1940-2010. Resour. Conserv. Recy. 2013, 73, 17-22.

Mao, J. S.; Dong, J.; Graedel, T. E. The multilevel cycle of anthropogenic lead: II. Results and discussion. Resour. Conserv. Recy. 2008, 52, 1050-1057.

Kavlak, G.; Graedel, T. Global anthropogenic tellurium cycles for 1940-2010. Resour. Conserv. Recy. 2013, 76, 21-26.

Graedel T. E.; Harper, E. M.; Nassar, N. T.; Nuss, P.; Reck, B. K. Criticality of metals and metalloids. P. Natl. Acad. Sci. USA, 2015, 112 (14), 4257-4262.

[10] Global Aluminium Recycling Committee (GARC). Global Aluminium Recycling Model. Global Aluminium Recycling Committee, International Aluminium Institute, London, 2007.

[11] Panousi, S.; Harper, E. M.; Nuss, P.; Eckelman, M. J.; Hakimian, A.; Graedel, T. E. Criticality of seven specialty metals. J. Ind. Ecol., 2015, 20, 837-853.

[12] Goonan, T. G., 2011, Vanadium recycling in the United States in 2004 (ver. 1.1, October 6, 2011), chap. S of Sibley, S.F., ed., Flow studies for recycling metal commodities in the United States: U.S. Geological Survey Circular 1196, p. S1-S7, available only on-line.

[13] Nuss, P.; Harper, E. M.; Nassar, N. T.; Reck, B. K.; Graedel, T. E. Criticality of iron and its principal alloying elements. Environ. Sci Technol., 2014, 48, 4171-4177.

[14] Ciacci, L.; Reck, B. K.; Nassar, N. T.; Graedel, T. E. Lost by design. Environ. Sci. Technol., 2015, 49 (16), 9443-9451.

[15] Donaldson, J. D.; Beyersmann, D. in Ullmann's Encyclopedia of Industrial Chemistry. Cobalt and Cobalt Compounds, Wiley-VCH Verlag GmbH \& Co. KGaA, 2000. 
1 [17] Harper, E. M.; Kavlak, G.; Burmeister, L.; Eckelman, M. J.; Erbis, S.; Sebastian Espinoza, V.; Nuss, P.; Graedel, T. E. Criticality of the geological zinc, tin, and lead family. J. Ind. Ecol., 2015, $19,628-644$.

4 [18] Meylan, G.; Reck, B. K. The anthropogenic cycle of zinc: status quo and perspectives. Resour.

Conserv. Recy., 2016, DOI: 10.1016/j.resconrec.2016.01.006

[19] Graf, G. G. in Ullmann's Encyclopedia of Industrial Chemistry. Zinc. (Wiley-VCH Verlag GmbH \& Co. KGaA, 2000).

[20] Thomas, D. W.; Mahmood, T.; Lindhal, C. B. in Kirk-Othmer Encyclopedia of Chemical Technology. Germanium and Germanium Compounds. John Wiley \& Sons, Inc., 2000.

[21] Grund, S. C.; Hanusch, K.; Wolf, H. U. in Ullmann's Encyclopedia of Industrial Chemistry. Arsenic and Arsenic Compounds, Wiley-VCH Verlag GmbH \& Co. KGaA, 2000.

[22] Nassar, N. T.; Barr, R.; Browning, M.; Diao, Z.; Friedlander, E.; Harper, E. M.; Kavlak, G.; Kwatra, S.; Jun, C.; Warren, S.; Yang, M-Y.; Graedel, T. E. Criticality of the geological copper family. Environ. Sci. Technol., 2012, 46 (2), 1071-1078.

[23] Kavlak, G.; Graedel, T. E.Global anthropogenic selenium cycles for 1940-2010. Resour. Conserv. Recy., 2013, 73, 17-22.

[24] Langner, B. E. in Ullmann's Encyclopedia of Industrial Chemistry. Selenium and Selenium Compounds, Wiley-VCH Verlag GmbH \& Co. KGaA, 2000.

[25] Du, X.; Graedel, T. E. Global in-use stocks of the rare earth elements: a first estimate. Environ. Sci. Technol., 2011, 45 (9), 4096-4101.

[26] Harper, E. M.; Diao, Z.; Panousi, S.; Nuss, P.; Eckelman, M. J.; Graedel, T. E. The criticality of four nuclear energy metals. Resour. Conserv. Recy. 2015b, 95, 193-201.

[27] Nassar, N. T. Anthropogenic losses of platinum group elements. In Elemental Recovery and

[28] Schulte-Schrepping, K.-H.; Piscator, M. in Ullmann's Encyclopedia of Industrial Chemistry.

[29] Psuja, D.; Hreniak, D.; Strek, W. Synthesis and characterization of indium-tin oxide

[30] Xu, H.; Zhu, G.; Zhou, H.; Yu, A. Preparation of monodispersed tin-doped indium oxide powders

[31] Al-Dahoudi, N.; Bisht, H.; Göbbert, C.; Krajewski, T.; Aegerter, M. A. Transparent conducting, anti-static and anti-static-anti-glare coatings on plastic substrates. Thin Solid Films 2001, 392, 299-304.

[32] Graf, G. G. in Ullmann's Encyclopedia of Industrial Chemistry. Tin, Tin Alloys, and Tin 35 Compounds, Wiley-VCH Verlag GmbH \& Co. KGaA, 2000. 
1 [33] Grund, S. C.; Hanusch, K.; Breunig, H. J.; Wolf, H. U. in Ullmann's Encyclopedia of Industrial

2 Chemistry. Antimony and Antimony Compounds, Wiley-VCH Verlag GmbH \& Co. KGaA, 2008.

[34] Greenwood, N. N.; Earnshaw, A. Chemistry of the Elements (2nd ed.), 581-582, ButterworthHeinemann, 1997.

[35] Audet, N.; Levitcharsky, B. Cadmium telluride production process. (Google Patents, 2010).

[36] Fthenakis, V. M. Life cycle impact analysis of cadmium in CdTe PV production. Renew. Sust. Energ. Rev. 2004, 8, 303-334.

[37] Nielsen, R. H.; Wilfing, G. in Ullmann's Encyclopedia of Industrial Chemistry. Hafnium and Hafnium Compounds, Wiley-VCH Verlag GmbH \& Co. KGaA, 2000.

[38] Lassner, E.; Schubert, W.-D.; Lüderitz, E.; Wolf, H. U. in Ullmann's Encyclopedia of Industrial Chemistry. Tungsten, Tungsten Alloys, and Tungsten Compounds, Wiley-VCH Verlag GmbH \& Co. KGaA, 2000.

[39] Carr, D. S.; Spangenberg, W. C.; Chronley, K.; Meshri, D. T. in Kirk-Othmer Encyclopedia of Chemical Technology. Lead Compounds, John Wiley \& Sons, Inc., 2000. 